\title{
AN APPLICATION OF REPRESENTATION THEORY TO PI-ALGEBRAS
}

\author{
JøRN B. OLSSON AND AMITAI REGEV ${ }^{1}$
}

\begin{abstract}
By realizing that the multilinear identities of degree $n$ of a PIalgebra form a left ideal in the group algebra $F\left[S_{n}\right]$, it is possible sometimes to use the representation theory of the symmetric group $S_{n}$ in the study of $T$ ideals and $P I$-algebras. In this note we demonstrate this method by proving: THEOREM. If the codimensions of a PI-algebra are bounded, then they are eventually bounded by 1 .
\end{abstract}

For basic definitions and notations, e.g., $F[X], V_{n}, Q_{n}$, codimension sequence, etc. we refer the reader to [6]. The correspondence

$$
x_{\sigma_{1}} \cdots x_{\sigma_{n}} \leftrightarrow\left(\begin{array}{c}
1 \cdots n \\
\sigma_{1} \cdots \sigma_{n}
\end{array}\right)=\sigma \in S_{n}
$$

extends to an isomorphism $V_{n} \approx F\left[S_{n}\right]$, where $F\left[S_{n}\right]$ is the group algebra of $S_{n}$ over $F$. We thus identify $V_{n}$ with $F\left[S_{n}\right]$. If $\sigma \in S_{n}$ and $f\left(x_{1}, \ldots, x_{n}\right) \in V_{n}$ are given, we consider $\sigma$ and $f$ as elements in the same group algebra $V_{n}$, so that the product $\sigma \cdot f$ is well defined. One verifies that

$$
\sigma \cdot f\left(x_{1}, \ldots, x_{n}\right)=f\left(x_{\sigma_{1}}, \ldots, x_{\sigma_{n}}\right) .
$$

It follows that for a $T$-ideal $Q, Q_{n}$ is a left (but generally not right) ideal of $V_{n}$. Since in characteristic 0 the multilinear identities completely determine all the identities of a $P I$-algebra, we shall assume $\operatorname{char} F=0$. It is well known that in this case $V_{n}$ is completely reducible over itself $[1, \S 15]$. Let $M$ be a (left) submodule of $V_{n}$. Define the length of $M, l(M)$, to be the number of irreducible components in a direct decomposition of $M$, and define the colength $l^{\prime}(M)$ to be $l^{\prime}(M)=l\left(V_{n}\right)-l(M)$, the length of a direct complement of $M$ in $V_{n}$. By the Krull-Schmidt theorem, this is well defined. For a given $T$ ideal $Q$, we thus obtain the sequence of colengths $l^{\prime}\left(Q_{n}\right)$.

Let us begin with a result from the representation theory of $S_{n}$.

For any integer $n>0$, it is well known that $S_{n}$ has exactly 2 irreducible representations of degree (dimension) 1, namely the unit and the sign representations. Denote by $a_{n}$ the minimal dimension of a nonlinear representation of $S_{n}$. We prove

Proposition 1. Let $n \geqslant 7$. Then

(i) $a_{n}=n-1$.

Received by the editors December 24, 1974.

AMS (MOS) subject classifications (1970). Primary 16A06, 16A38; Secondary $20 \mathrm{C} 30$.

${ }^{1}$ The second author was partially supported by NSF grant GP 28696.

( ) American Mathematical Society 1976 
(ii) There are exactly two irreducible representations of $S_{n}$ of degree $n-1$. They correspond to the partitions $(n-1,1)$ and $\left(2,1^{n-2}\right)$ of $n$.

Note. (i) fails only for $n=4$ and (ii) only for $n=6$.

Proof. We use basic facts from the representation theory of $S_{n}$. Let $\operatorname{Par}(n)$ denote the set of all partitions of $n$, i.e., sequences of integers $\lambda=\left(\alpha_{1}, \ldots, \alpha_{k}\right)$, satisfying $\alpha_{1} \geqslant \alpha_{2} \geqslant \cdots \geqslant \alpha_{k}$ and $\alpha_{1}+\cdots+\alpha_{k}=n$. For $\lambda \in \operatorname{Par}(n)$ we associate the Young diagram, defined in [2, p. 20], which we denote by $Y(\lambda)$. To a given $\lambda \in \operatorname{Par}(n)$ there is a canonical way to associate an irreducible representation $[\lambda]$ of $S_{n}$ [2, pp. 60-63]. Our main tool is the Branching Theorem (B.T.) [2, p. 85], which describes the decomposition into irreducible components of the restriction $\left.[\lambda]\right|_{S_{n-1}}$ of $[\lambda]$ to $S_{n-1}$ in terms of Young diagrams. We start by

Lemma 2. Let $\lambda \in \operatorname{Par}(n), n \geqslant 5$. Assume that $\operatorname{deg}[\lambda]=f_{\lambda}>1$. If $\left.[\lambda]\right|_{S_{n-1}}$ is irreducible, then $\left.[\lambda]\right|_{S_{n-2}}$ does not have a linear irreducible component.

Proof. Suppose not. Since $\left.[\lambda]\right|_{S_{n-1}}$ is irreducible, the B.T. implies that $Y(\lambda)$ is rectangular. Therefore, there exists a factorization $n=k \cdot l$, such that $\lambda=\left(k^{l}\right)$. We assume that $\left.[\lambda]\right|_{S_{n-2}}$ has a linear component. Since the linear representations of $S_{n-2}$ correspond to the partitions $\left(1^{n-2}\right)$ and $(n-2)$, B.T. implies that $k=l=2$. This is because removing 2 squares in the bottom right-hand corner of $Y(\lambda)$ should give $Y\left(1^{n-2}\right)$ or $Y(n-2)$. Thus $n=k \cdot l$ $=4$, a contradiction.

We now continue the proof of Proposition 1 and apply induction. It is true for $n=7,8$ by the tables in [5, p. $265 \mathrm{ff}]$. We assume the result holds for $n-1 \geqslant 8$ and that there exist $\lambda \in \operatorname{Par}(n)$ such that $1 \leqslant f_{\lambda} \leqslant n-2$, where $f_{\lambda}=\operatorname{deg}[\lambda]$. By the induction hypothesis and B.T., either $f_{\lambda}=n-2$ and $\left.[\lambda]\right|_{S_{n-1}}$ is irreducible, or $\left.[\lambda]\right|_{S_{n-1}}$ decomposes into linear components. The latter is impossible, since then $A_{n}$-the alternating group-would be contained in the kernel of $[\lambda]$. Therefore $f_{\lambda}=n-2$. Again, by the induction hypothesis, $\left.[\lambda]\right|_{S_{n-2}}$ must have a linear component, contrary to Lemma 2. This proves (i).

Suppose $\lambda \in \operatorname{Par}(n), \lambda \neq(n-1,1),\left(2,1^{n-2}\right)$ and $f_{\lambda}=n-1$. B.T. implies then that $\left.[\lambda]\right|_{S_{n-1}}$ is irreducible, and the induction hypothesis for $n-2$ implies that $\left.[\lambda]\right|_{S_{n-2}}$ has a linear component, again contradiction Lemma 2.

REMARK. One can define the next sequence of degrees of representations, etc., and try to compute or give an asymptotic estimate to them. This has been done by R. Rasala.

We will also need

Proposition 3. Let $Q$ be a T-ideal generated by polynomials of degree $\leqslant d$. Let $n \geqslant d$. Then

$$
Q_{n+1}=T\left(Q_{n}\right) \cap V_{n+1} .
$$

Here $T\left(Q_{n}\right)$ is the $T$-ideal generated by the polynomials of $Q_{n}$.

Proof. Since char $F=0$, we may assume that $Q$ is generated by multilinear polynomials. (See Lemma (4.1) in [4].) Denote $\bar{Q}_{n+1}=T\left(Q_{n}\right) \cap V_{n+1}$. Obviously $\bar{Q}_{n+1} \subseteq Q_{n+1}$. Now $Q_{n+1}$ is linearly spanned over $F$ by multilinear polynomials of the form $a f\left(M_{1}, \ldots, M_{r}\right) b$, where $f\left(x_{1}, \ldots, x_{r}\right)$ is among the multilinear generators of $Q(r \leqslant d)$ and $a, b, M_{1}, \ldots, M_{r}$ are monomials. We 
show that such a polynomial is in $\bar{Q}_{n+1}$. If $a \neq 1$, assume $a=x_{n+1} a^{\prime}$. Then $a^{\prime} f\left(M_{1}, \ldots, M_{r}\right) b \in Q_{n}$, so $x_{n+1} a^{\prime} f\left(M_{1}, \ldots, M_{r}\right) b \in \bar{Q}_{n+1}$. The same argument can be used if $b \neq 1$, so assume $a=b=1$.

Since $f\left(M_{1}, \ldots, M_{r}\right)$ is multilinear of degree $n+1$ and $n+1>d \geqslant r$, it follows that at least one of the monomials $M_{i}$ have degree at least 2 . We may assume without loss of generality that $M_{1}=M_{1}^{\prime} x_{n} x_{n+1}$. Then

$$
f\left(M_{1}^{\prime} x_{n}, M_{2}, \ldots, M_{r}\right) \in Q_{n},
$$

and we conclude again, that $f\left(M_{1}^{\prime} x_{n} x_{n+1}, M_{2}, \ldots, M_{r}\right) \in \bar{Q}_{n+1}$.

We are now going to prove the main theorem after a sequence of preliminary results.

In the following, $\left\{c_{n}\right\}$ is the sequence of codimensions for a $T$-ideal $Q$. $\left(c_{n}=c_{n}(Q)\right.$.)

Lemma 4. If $c_{n} \leqslant k$ for all $n$, then $c_{n} \leqslant 2$ for $n \geqslant k+1$.

Proof. Let $Q_{n}=Q \cap V_{n}$. Write $V_{n}=Q_{n} \oplus J_{n}$ (as $V_{n}$ left modules). Obviously, $c_{n}=\operatorname{dim} J_{n}$. Decompose $J_{n}$ as a direct sum of irreducible $V_{n}$-left modules. At most two of the components have dimension 1, and the others have dimension $\geqslant n-1$ by Proposition 1 . Therefore, if $n \geqslant k+1$, there can be only linear components in $J_{n}$, and $c_{n} \leqslant 2$.

Lemma 5. (1) If $c_{N}=0$, then $c_{n}=0$ for all $n \geqslant N$. (2) Assume $c_{n} \neq 0$ for all $n$. If $c_{N}=1$, then $c_{n}=1$ for all $n \geqslant N$.

Proof. (1) $c_{N}=0$ implies $Q_{N}=V_{N}$. Then obviously $Q_{n}=V_{n}$ for all $n \geqslant N$.

(2) Assume $c_{N}=1$. In the previous notation, $V_{N}=Q_{N} \oplus J_{N}$. There are exactly two one-dimensional left-submodules of $V_{n}$, generated by the standard polynomial $s_{N}=\sum_{\sigma \in s_{N}}(-1)^{\sigma} \sigma$ and the unitary polynomial $u_{N}=\sum_{\sigma \in s_{N}} \sigma$.

We claim that $J_{N}=F u_{N}$. If not, then $u_{N} \in Q_{N}[1, \S 25]$. As a polynomial, $u_{N}=\sum_{\sigma} x_{\sigma_{1}} \cdots x_{\sigma_{N}}$. By substituting $x_{i} \rightarrow x$ for all $i, u_{N}$ implies $x^{N}$. But then the Nagata-Higman theorem implies that for $l=2^{N}-1, Q_{l}=V_{l}$, so $c_{l}=0$, contradicting our assumption.

We conclude that $J_{N}=F u_{N}$. Therefore $Q_{N}=C_{N}$, where $C=T([x, y])$. By Proposition 3, we get $Q_{n}=C_{n}$ for $n \geqslant N$, so $c_{n}=1$ for $n \geqslant N$.

To prove the main theorem we need only exclude that $c_{n}$ is eventually equal to 2 .

Lemma 6. Let $d \geqslant 2$ and $P=T\left(\left[x_{1} \cdots x_{d}, x_{d+1}\right]\right)$, and let $\left\{h_{n}\right\}$ be the codimensions. Then $h_{n}=1$, if $n \geqslant d+2$.

Proof. Assume $n \geqslant d+2$. If $\sigma \in S_{n}$, let $M_{\sigma}=x_{\sigma_{1}} \cdots x_{\sigma_{n}}$. Define an equivalence relation on the elements of $S_{n}$ by

$$
\sigma \sim \rho \Leftrightarrow M_{\sigma}-M_{\rho} \in P_{n} .
$$

Now $G=\left\{\sigma \in S_{n} \mid \sigma \sim(1)\right\}$ is a subgroup of $S_{n}$, because if $\sigma, \rho \in G$, then

$$
M_{\sigma \rho}=\sigma M_{\rho} \equiv \sigma M_{(1)}=M_{\sigma} \equiv M_{(1)} \quad\left(\bmod P_{n}\right)
$$

so $\sigma \rho \in G$.

We want to show $G=S_{n}$ and to do this we need only show $(k, k+1) \in G$ 
for $k=1,2, \ldots, n-1$. Since $n-2 \geqslant d$, we get, modulo $P_{n}$,

$$
\begin{aligned}
x_{1} \cdots x_{n} & \equiv x_{n} x_{1} \cdots x_{n-1} \\
& \equiv \cdots \\
& \equiv x_{k} x_{k+1} \cdots x_{n} x_{1} \cdots x_{k-1} \equiv x_{k} x_{k+2} \cdots x_{n} x_{1} \cdots x_{k-1} x_{k+1} \\
& \equiv x_{k+2} \cdots x_{n} x_{1} \cdots x_{k-1} x_{k+1} x_{k} \\
& \equiv \cdots \\
& \equiv x_{1} \cdots x_{k-1} x_{k+1} x_{k} x_{k+2} \cdots x_{n}
\end{aligned}
$$

for any $k, 1 \leqslant k \leqslant n-1$, whence $(k, k+1) \in G$.

This shows $G=S_{n}$, so $h_{n} \leqslant 1$. But $T\left(\left[x_{1} \cdots x_{d}, x_{d+1}\right]\right) \subseteq T([x, y])=C$, and $C$ has constant codimensions 1 . Therefore $h_{n}=1$.

Let us remark:

Lemma 7. Let $v=\sum_{\sigma} \alpha_{\sigma} \sigma \in V_{n}, \alpha_{\sigma} \in F$. Then

$$
s_{n} \in V_{n} v \Leftrightarrow \sum_{\sigma}(-1)^{\sigma} \alpha_{\sigma} \neq 0, \quad u_{n} \in V_{n} v \Leftrightarrow \sum \alpha_{\sigma} \neq 0 .
$$

This is because $s_{n} v=\left(\sum_{\sigma}(-1)^{\sigma} \alpha_{\sigma}\right) s_{n}$ and $u_{n} v=\left(\sum_{\sigma} \alpha_{\sigma}\right) u_{n}$.

Corollary 8. Let $v=\left[x_{1} \cdots x_{d}, x_{d+1}\right]=(1)-(1,2, \ldots, d+1)$. If $d$ is even, then $u_{d+1} \notin V_{d+1} v$ and $s_{d+1} \notin V_{d+1} v$.

Proof of MAIN THEOREM. By a previous remark, if $Q$ is a counterexample to the theorem, then the codimensions are eventually 2 . Suppose $c_{n}=2$ for $n \geqslant N$. Then for $n \geqslant N, V_{n}=Q_{n} \oplus J_{n}$, where $J_{n}=F u_{n} \oplus F s_{n}$. Let $n \geqslant N$ be odd. Then $\left[x_{1} \cdots x_{n-1}, x_{n}\right] \in Q_{n}$ by Corollary 8 , so $P=T\left(\left[x_{1} \cdots x_{n-1}, x_{n}\right]\right)$ $\subseteq Q$. If $\left\{h_{k}\right\}$ are the codimensions for $P$, then by Lemma $6, h_{k}=1$ for $k \geqslant 2 n+1$. Obviously, $h_{k} \geqslant c_{k}$ for $k \geqslant n$, which is a contradiction.

Note. From the proof it follows that if $c_{n} \leqslant m$ for all $n$, then $c_{n} \leqslant 1$ for $n \geqslant m+3$. Also, for $n$ sufficiently large, $Q_{n}=V_{n}$ or $Q_{n}=C_{n}$, where $C=T([x, y])$.

It is possible to combine information about codimensions with Proposition 1 to estimate the colength sequence. Using the technique of Lemma 4 , it is easy to show

Proposition 9. Let $c_{n}$ be the codimensions, $l_{n}^{\prime}$ the colengths of a T-ideal. If $c_{n} \leqslant(k+1) n-k$, where $k \geqslant 0$ is an integer, then $l_{n}^{\prime} \leqslant k+2$. In particular, if $c_{n} \leqslant n$, then $l_{n}^{\prime} \leqslant 2$.

Note. If $c_{n} \leqslant n$ for all $n$, then eventually either $c_{n}=n, c_{n}=1$ or $c_{n}=0$. This follows from Proposition 1, Lemma 5 and the fact that, if $c_{k}=k-1$ for some $k \geqslant 5$, then eventually $c_{n}=0$. (If $c_{k}=k-1$, then $F u_{k} \subseteq Q_{k}$.)

We conclude by giving two examples where $c_{n} \leqslant n$.

1. Let $f(x)$ be a polynomial of degree 3 which is not an identity for the Grassmann algebra $E . Q=T([[x, y], z])$ is the ideal of identities of $E$, [4]. Let $P=T([[x, y], z], f)$. It was proved (unpublished) by the second author that $c_{n}(P) \leqslant n$.

2. Let $Q=T([x, y] \cdot z)$. Then $c_{n}(Q)=n$, [3]. In this case, $l_{n}^{\prime}=2$ for all $n \geqslant 2$. 
Final Remarks. 1. An alternative proof of Proposition 1(i) can be found in [*], as an appendix to that book.

2. After finishing this paper, the authors learned from Professor D. S. Passmann that he is able to prove our main theorem for arbitrary characteristic, provided that the $P I$-algebra has an identity element. This approach to the problem is quite different from ours.

\section{REFERENCES}

1. C. Curtis and I. Reiner, Representation theory of finite groups and associative algebras, Pure and Appl. Math., vol. 11, Interscience, New York and London, 1962. MR 26 \#2519.

2. A. Kerber, Representations of permutation groups. I, Lecture Notes in Math., vol. 240, Springer-Verlag, Berlin and New York, 1971. MR 48 \#4098.

3. A. A. Klein and A. Regev, The codimensions of a PI-algebra, Israel J. Math. 12 (1972), 421-426. MR 48 \#4020.

4. D. Krakowski and A. Regev, The polynomial identities of the Grassmann algebra, Trans. Amer. Math. Soc. 181(1973), 429-438. MR 48 \#4005.

5. D. E. Littlewood, The theory of group characters and matrix representations of groups, Oxford Univ. Press, Oxford, 1958.

6. A. Regev, Existence of identities in $A \otimes B$, Israel J. Math. 11 (1972), 131-152. MR 47 \#3442.

*. W. Burnside, Theorem of groups of finite order, 2nd ed., Cambridge, 1911

Department of Mathematics, University of Chicago, Chicago, Illinois 60637

Mathematische Abteilung, Universität Dortmund, 46-Dortmund-Hombruch, West GeRMANY 